\title{
Is "benign intracranial hypertension" really benign?
}

\author{
S G BARBER AND N GAR VA N \\ From the Department of Medicine, General Hos pital, Birmingham, and the Department of \\ Neurosurgery, Midland Centre for Neurology and Neurosurgery, Smethwick
}

SUMMARY Hypothalamic-hypophyseal insufficiency has been found in seven of eight patients with so-called benign intracranial hypertension, of whom four showed an inadequate adrenal response to stress. The syndrome of benign intracranial hypertension cannot therefore be considered entirely benign and patients should receive full endocrinological assessment and follow up.

The development of sensitive and specific tests of hypothalamic and pituitary function ${ }^{1}$ has enabled the mechanisms of pituitary infarction to be re-examined. The ability to monitor intracranial pressures led to the detection of bursts of intracranial hypertension in "normal pressure" hydrocephalus ${ }^{2}$ which have been implicated as the cause of hypothalamic hypophyseal damage in such patients. ${ }^{34}$ Since the intracranial pressure in patients with the syndrome of benign intracranial hypertension may be severe enough to cause blindness in addition to papilloedema, ${ }^{5}$ we decided to assess the endocrine status of such patients.

\section{Patients, Materials and Methods}

Nine patients aged $24-55$ years (mean 41.9 years) were diagnosed as having benign intracranial hypertension on the basis of history, physical examination, and the absence of demonstrable pathology to explain the raised intracranial pressure. One patient was male and the others female. Anterior pituitary function was assessed in response to insulin-induced hypoglycaemia, thyrotrophin-releasing hormone (TRH), and gonadotrophin-releasing hormone (GnRH) after an overnight fast. ${ }^{1}$ Basal samples were taken for rcutine blood count, biochemical profile, thyroxine, T3 uptake test, and either oestradiol or total androgen (depending on the sex of the

Address for reprint requests: Dr SG Barber, The General Hospital, Steelhouse Lane, Birmingham B4 6NH.

Accepted 15 August 1979 patient). All hormone assays were performed subject to the quality control criteria of the supraregional assay service, using methods described elsewhere. ${ }^{6}$ Blood sugar estimations were performed by the ferricyanide method of Technicon. ${ }^{7}$ All blood samples were taken via an indwelling forearm venous cannula. In the absence of suggestive symptoms, posterior pituitary function was not tested.

\section{Results}

Basal thyroid function (thyroxine and T3 uptake) tests were normal in all patients. The results of other endocrine tests are shown in the table. Only two patients (cases 2 and 6) had received steroids at any time and in the former they had not been given for six months before testing. The latter patient was on dexamethasone at the time of testing and this probably explains the blunted, sustained TSH response to TRH.

The cortisol response to insulin-induced hypoglycaemia was abnormal in five patients (cases 1 , $4,7,8$, and 9) and was not measured in the patient receiving dexamethasone. Although the basal resting cortisol levels were within normal limits, the response to stress was inadequate. ${ }^{8}$ All patients experienced definite symptoms of hypoglycaemia (see table). Growth hormone responses to the same hypoglycaemic stimulus were abnormal in five patients (cases $2,4,6,7$, and 8) although a rise from undetectable levels to $18 \mathrm{mU} / 1$ in case 6 probably represented "normal" function for a patient receiving steroids.

The luteinising hormone (LH) response to GnRH was abnormally sustained in three patients 
(cases 1, 2, and 4). In four patients (cases 2, 4, 7, and 8) the high basal levels of LH and FSH were compatible with their postmenopausal status. Basal prolactin levels were abnormal in one patient (case 5) with a rise to 3350, 3350, 2450, and $2950 \mathrm{mU} / \mathrm{l}$ at $30,60,90$, and 120 minutes after TRH respectively. In the absence of a detectable pituitary tumour producing prolactin this also suggests hypothalamic damage.

Thus, of the eight patients not receiving corticosteroids at the time of testing, seven had abnormal pituitary responses to conventional testing and of these, four had an inadequate adrenocortical response to stress.

\section{Discussion}

Benign intracranial hypertension, pseudotumour cerebri, serous meningitis, and otitic hydrocephalus are names for a syndrome which is characterised by increased intracranial pressure for which intracranial mass lesions, obstruction of the cerebral ventricles, intracranial infection, hypertensive encephalopathy, and chronic carbon dioxide retention have been excluded and in which spontaneous recovery is usual. It may be associated with menstrual irregularity or amenorrhoea and has variously been reported as a complication of Addison's disease with improvement after replacement therapy ${ }^{9}$ and as being more likely to occur when steroid dosage is reduced and "relative adrenal insufficiency" is present. ${ }^{10}$ Our results show that hypothalamichypophyseal damage occurs in most patients with this condition. As early as 1926 Simonds et al ${ }^{11}$ assumed that raised intracranial pressure caused pituitary infarction in their patient who had a frontal glioma. This presumption was supported by finding hypothalamic and pituitary damage in animals subjected to artificially induced rises in intracranial pressure using balloons ${ }^{12}$ and in human patients after the transient bursts of intracranial hypertension found in so-called "normal pressure" hydrocephalus where all six patients tested had abnormal results. ${ }^{34}$ Therefore the endocrine disturbances in our patients were probably like the papilloedema and blindness, secondary to the rise in intracranial pressure. Such hypertension may cause herniation of the diaphragma sellae into the sella turcica ${ }^{13}$ with subsequent disturbance in pituitary function. Our patients showed no radiological evidence of the "empty sella syndrome" and the endocrine results suggest hypothalamic rather than pituitary damage. Moreover, the galactorrhoea which has occasionally been associated with benign intra- 
cranial hypertension ${ }^{6}$ may be caused by hypothalamic damage leading to inadequate production of prolactin-release-inhibiting factor.

The difficulties in interpreting the results in patients receiving steroids are exemplified in case 6, where the TSH and growth hormone responses may have been due either to the steroids or to local damage, and the ACTH-adrenal response to stress could not be tested. In the light of the other results, patients such as these need reassessing after cautious withdrawal of steroids, and replacement therapy with steroids should be given for trauma or illness pending such reassessment.

So-called benign intracranial hypertension may not be completely benign and patients with this diagnosis should be assessed and followed up endocrinologically. Assessment should be performed after cessation of steroid therapy if this has been given, and if patients have an inadequate cortisol response to stress, replacement therapy should be started, as for other patients with adrenal insufficiency.

We thank the various physicians, neurologists and neurosurgeons who allowed us to examine their patients; Professor Butt, Midland Endocrine Laboratory, Women's Hospital, Birmingham, and Dr Northam, General Hospital, Birmingham, for endocrine and biochemical estimations; Hoechst Pharmaceuticals, who supplied the gonadotrophinreleasing hormone; and, of course, the patients.

\section{References}

1 Harsoulis P, Marshal JC, Juka SF, et al. Combined test for assessment of anterior pituitary function. $\mathrm{Br}$ Med $J$ 1973; 4:326-9.
2 Symon L, Dorsch NWC, Stephens RJ. Pressure waves in so-called low pressure hydrocephalus. Lancet 1972; 2:1291-2.

3 Barber SG, Garvan N. Hypothalamic hypopituitarism in a patient with normal pressure hydrocephalus. $\mathrm{Br}$ Med J 1978; 1:817-8.

4 Barber SG, Garvan N. Hypothalamic hypopituitarism in normal pressure hydrocephalus. Lancet 1978; 2:1079.

5 Fishman RA. In Beeson PB, McDermott W (eds). Textbook of Medicine. 14th ed, WB Saunders, Philadelphia: 1975: chap. 10.

6 Kandeel FR, Butt WR, London DR, Lynch SS, Logan Edwards R, Rudd BT. Oestrogen amplification of LR-RH response in the polycystic ovary syndrome and response to clomiphene. Clin Endocrinol 1978; 9:429.

7 Hoffman WS. A rapid photoelectric method for the determination of glucose in blood and urine. J Biol Chem 1937; 120:51.

8 Hall R, Anderson J, Smart GA, et al. Fundamentals of Clinical Endocrinology. 2nd ed. London: Pitman Medical, 1974.

9 Greer M. Benign Intracranial Hypertension. Neurology (Minneap) 1964; 14:668.

10 Neville BGR, Wilson J. Benign intracranial hypertension following corticosteroid withdraval in childhood. Br Med $J$ 1970; 3:554.

11 Simmonds JP, Brands WW. Studies in pathologic anatomy of the hypophysis. IV. Necrosis and atrophy. Arch Path 1926; 2:18-22.

12 Nakajima T, Kageyama N, Kurimoto $\mathrm{T}$, et al. Pathological changes and microcirculatory disturbances in both hypophysis and hypothalamus at the time of increased intracranial pressure. In Procee'ings of the Annual Meeting of the Japanese Endocrinology Society, 1971, p.2.

13 Brisman T, Hughes JEO, Mount LA. Cerebrospinal fluid, rhinorrhoea and the "empty sella". J Neurosurg 1969; 31:538-43. 